\title{
Modeling turbulent stream flows over rough permeable beds
}

\author{
G. Rousseau, I. Pascal \& C. Ancey \\ Laboratory of Environmental Hydraulics, Department of Civil Engineering. Ecole Polytechnique Fédérale \\ de Lausanne (EPFL), Lausanne, Switzerland
}

\begin{abstract}
In steep streams, turbulent flows run over coarse sediments that are similar in size to flow depth. Under these conditions, a significant part of the flow may seep through the permeable bed. Based on experimental data, this paper presents a model able to reproduce the vertical structure of flows over rough permeable beds in low relative submergence conditions. Experiments were performed on open-channel flows passing over tilted coarse-grained beds with slopes within the $0.5 \%-4 \%$ range. Fluid velocities were measured by Particle Image Velocimetry (PIV) and a technique called Refractive Index-Matched Scanning (RIMS), allowing the interior of the bed to be examined. By applying the double averaging methodology, porosity and mean velocity, as well as turbulent and dispersive stresses profiles were collected from the subsurface to the free surface. A turbulent boundary layer over the rough bed was observed while experiments were run at intermediate Reynolds numbers, i.e. $R e=O(1000)$. Under these flow conditions, viscosity played a non-negligible role through the van Driest damping effect. Based on the Prandtl mixing length theory, we propose a model for the turbulent stress that takes into account the continuous porosity profile, damping and dispersive effects. Finally, we show a good agreement between the model and classic flow resistance laws employed for river studies. Our model contrasts with existing boundary-layer models which generally assume a discontinuous porosity profile at bed interface, whether the bed is permeable or impermeable.
\end{abstract}

Keywords: Turbulent boundary layers, low relative submergence, steep streams, permeable beds

\section{INTRODUCTION}

Steep streams exhibit distinctive features unshared by lowland rivers: (i) their flow-depth and roughness scales are similar; and (ii) their bed permeability is much higher. Both features explain why predicting flow resistance or the threshold of incipient motion is more complicated for mountain streams than for lowland rivers.

These flows may advantageously be analyzed through the prism of the double averaging framework. Considering a flow volume parallel to the bed (as shown in Figure 1), the double averaging method consists of averaging over the temporal scale (this is the classic Reynolds averaging step), and then averaging is performed over the spatial scale of a thin layer parallel to the bed (Nikora et al. (2007)). The stream-wise length scale $L$ on which spatial averagings are made to obtain quasi-uniform conditions defines the mesoscopic scale.

As observed in Figure 1, applying spatial averaging to naturally shaped beds results in smooth porosity profiles, with porosities increasing from bed porosity $\epsilon_{b}$, to a porosity equal to 1 in the surface layer above the roughness crest $z_{r c}$. When bed roughness is similar in size to flow depth, i.e in case of low relative submergence, a non negligible part of the flow seeps trough the roughness and subsurface layers. Additionnaly, the flow depth definition $\left(h_{f}=z_{\text {surf }}-z_{\text {bed }}\right)$ is not univocal and an arbitrary origin must be chosen for $z_{b e d}$ between $z_{r c}$ and the troughs of the roughness elements $z_{t}$. As highlighted by Pokrajac et al. (2006), this definition problem is essential in low relative submergence conditions. It affects values taken by quantities such as the shear velocity $u^{*}=\sqrt{g h_{f} i}$ or the Shields number $S h=h_{f} i /\left(\left(\rho_{s} / \rho_{f}-1\right) d_{p}\right)$, where $g$ denotes gravity acceleration, $d_{p}$ is the grain diameter, $i$ is bed slope, and $\rho_{f}$ and $\rho_{s}$ are the fluid and grain 


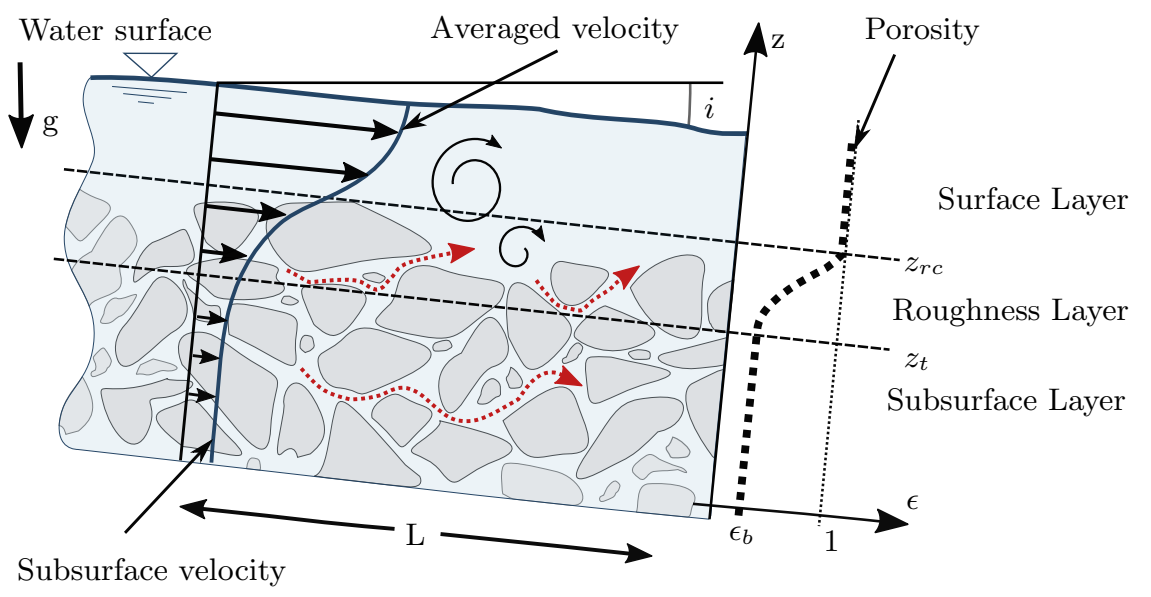

Figure 1. Grain-scale processes of a turbulent flow over a rough permeable bed and the double averaging procedure. The red dotted arrows represent streamlines through the roughness and subsurface layers forming the permeable bed.

densities respectively. The apparent lack of universal defintion for $h_{f}$ for low relative submergence flows warrants great care in establishing new findings.

Analyzing flow over rough beds at the mesoscopic scale by employing the double averaging technique is now well-established (e.g. Mignot et al. 2009, Voermans et al. 2017). In terms of spatial resolution, this mesoscopic scale is located mid way between fine descriptions of flows at pore scales (using Direct Numerical Simulations or Large Eddy Simulations for instance) and depth-averaged approach. Fine descriptions may seem more powerful, but are computationally expensive when applied to real-world scenarios at the river scale, while the depth-averaged approach requires empirical equations relating depth-averaged quantities. Owing to its low computational cost, the depth averaged approach has been widely deployed for predicting river flows in the last decades but still remains unable to reproduce documented observations in steep streams. A typical example is provided by flow resistance, whose modeling in the shallow-water equations generally relies on the assumption of a logarithmic velocity profile, which is valid only for large submergence conditions. Recent studies have shown the large bias in the bed shear stress value when applying this assumption to steep streams (Rickenmann \& Recking 2011). Authors suggested using an empirical power-law function fitted to field data. Not only do empirical flow resistance equations improve predictions, but they also show that the classic physical picture underpinning the log-profile assumptions breaks down when looking at flows exhibiting a small relative submergence.

In this paper, we study flows over rough permeable bed under low submergence conditions, with a focus on modeling the vertical distribution of flow variables at the mesoscopic scale. After experimental validation, determining vertical velocity profiles makes it possible to express flow resistance as a function of depth-averaged variables, and then compare it with empirical flow resistance equations found in literature (e.g. Rickenmann \& Recking (2011) for a review).

Obtaining double-averaged profiles in steep streams is challenging, and this explains the scarcity of experimental results to date. In his PhD thesis, Rousseau (2019) devised an experimental setup to acquire new data and test various modeling assumptions. In this proceeding paper, we summarize the experimental work and the model based on the double-averaged momentum equation. Further details on the experimental procedure can be found in Rousseau \& Ancey (2020). In the results part, the model is compared with experiments and well-known flow resistance equations used in coarse-grained river studies.

\section{EXPERIMENTAL WORK}

\subsection{Setup}

Experiments were performed in a 6-cm-wide tilting flume, as depicted in Figure 2. A constant head tank provided a steady fluid discharge into the system. Equal proportions of borosilicate beads of two diameters ( 7 and $9 \mathrm{~mm}$ ) were randomly packed into the flume bottom, forming the 


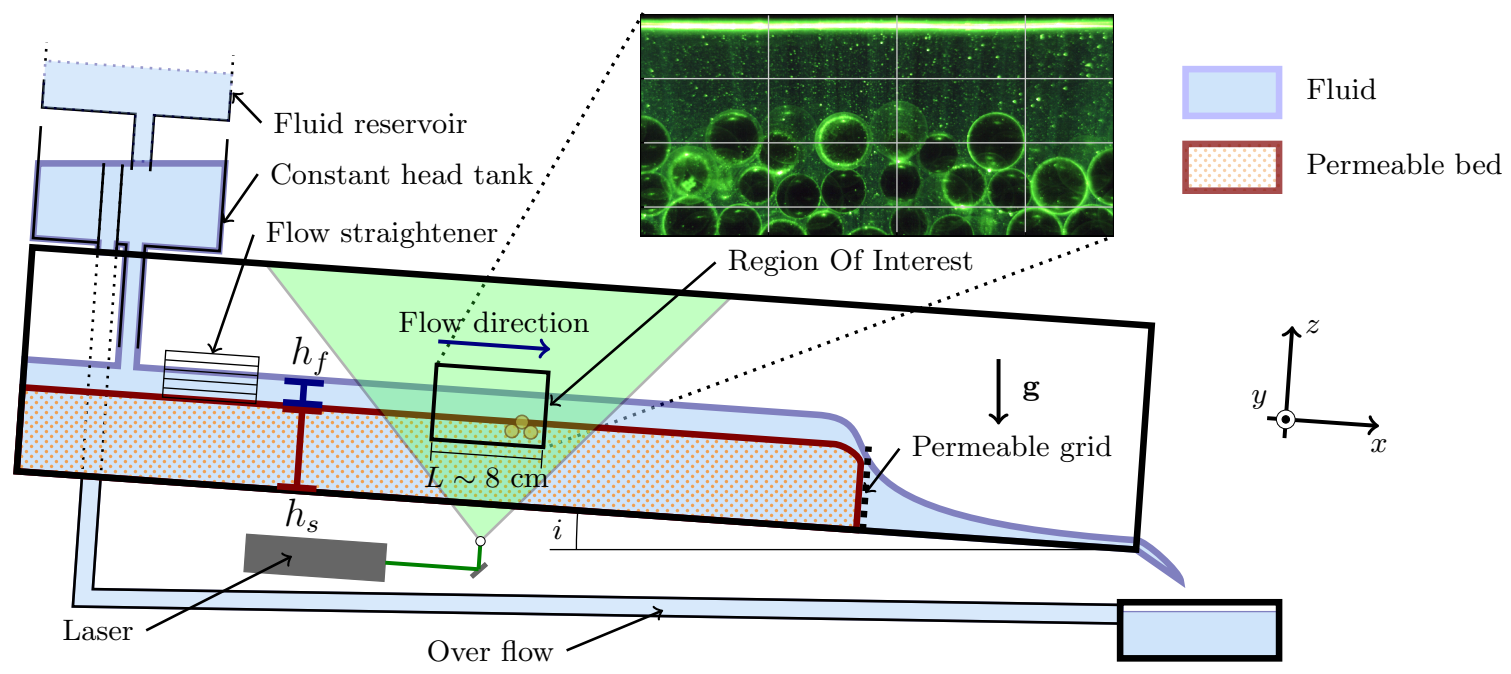

Figure 2. Sketch of the experimental set-up

coarse-grained bed. The median diameter was $d_{p}=d_{50}=8 \mathrm{~mm}$. A bimodal size distribution has been used because otherwise the beads arrange itself in structured parallel layers causing undesirable bias in the averaged porosity and velocity profiles (as observed by Ni \& Capart (2015)). Before each run, the upper layer was flattened out to form a uniform bed of height $h_{s}=5 \mathrm{~cm}$.

The Refractive Index-Matched (RIM) technique has been employed for locating beads and measuring interstitial velocities. This involves matching the fluid refractive index with that of the glass beads, which makes it possible to access the information about beads location and interstitial flow velocities. The iso-index fluid was prepared by mixing volumic concentrations of $60 \%$ benzyl alcohol and $40 \%$ ethanol (BAE). Fluid and sediment characteristics were measured (see Table 1).

Table 1. Fluid and sediment characteristics measured at $20^{\circ} \mathrm{C}$

\begin{tabular}{ccccccc}
\hline$i(\%)$ & $n_{f} *$ & $d_{p}(\mathrm{~mm})$ & $h_{s}(\mathrm{~cm})$ & $\rho_{s}\left(\mathrm{~kg} \mathrm{~m}^{-3}\right)$ & $\rho_{f}\left(\mathrm{~kg} \mathrm{~m}^{-3}\right)$ & $\nu\left(\mathrm{mP} \mathrm{s}^{2}\right)$ \\
\hline $1.0-4.0$ & 1.472 & 8.0 & 5.0 & 2200 & 950 & 3.0 \\
\hline
\end{tabular}

*fluid refractive index

Compared to other materials used in RIM techniques, combining borosilicate, ethanol and benzyl alcohol leads to mixtures whose relative density between solid and fluid is close to that found in rivers. Meeting similarity criteria such as the Shields and Froude numbers was necessary to obtain flow conditions that mimicked those encountered in real-world scenarios. If we had followed Ni \& Capart (2015) and used a sediment of polymethyl methacrylate (PMMA), with a density of $\rho_{s}=1190 \mathrm{~kg} \cdot \mathrm{m}^{-3}$, it would have been impossible to conduct experiments on steep slopes without observing sediment transport. The same problem would have occur if we had employed a popular RIM fluid mixture made of NaI (e.g. as in Voermans et al. (2017)) since, in this case, the fluid has an unexpectedly high density: $\rho_{f, \mathrm{NaI}}=1770 \mathrm{~kg} \cdot \mathrm{m}^{-3}$. In the present context, we needed a stable bed resisting to the stream's erosive action for supercritical flows.

\subsection{Velocimetry and scanning}

Using PIV, we seeded the flow with micrometric tracers lit up by a laser. In contrast with earlier PIV measurements using a fixed laser sheet, we scanned the flow across its width by moving laser sheet. This methodology is similar to Ni \& Capart (2015) and has been called PIV-RIMS (the S means Scanning). With this procedure we collected double-averaged profiles such as the porosity, the streamwise velocity $U_{x}$ as well as the turbulent $\tau_{t}$ and dispersive $\tau_{d}$ stresses (see Section 3 for 


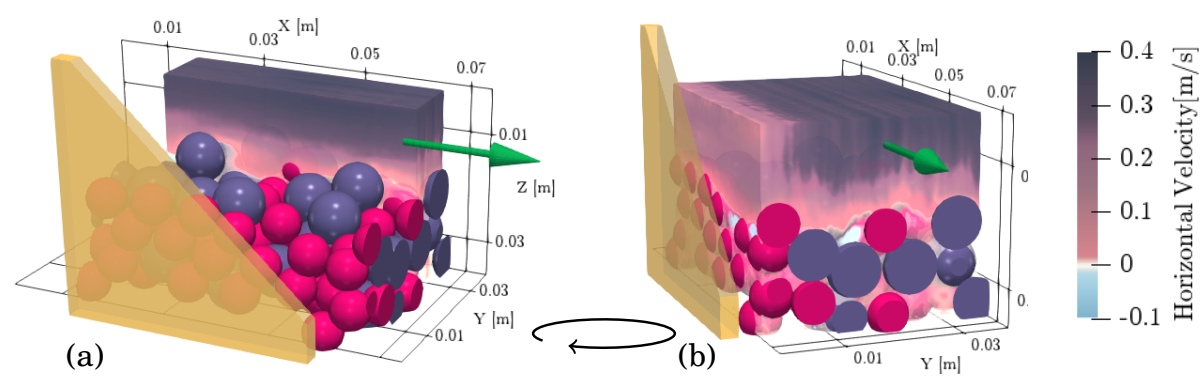

Figure 3. Three-dimensional visualization of the horizontal velocity. (a) Side view of a slab of the velocity field at $Y=25 \mathrm{~mm}$ from the sidewall. (b) Front view of the flow, sliced along $y$. This view enables us to appreciate the sidewall's influence. $Y$ is the distance from the sidewall. The origins of $Z$ and $X$ are arbitrary.

definitions). The PIV algorithm deducing the local displacement of features is available in open source from the public GitHub repository: https://github.com/groussea/opyflow (see the supplementary information in Rousseau (2019) - Annex C). The laser sheet's linear movement perpendicular to the flow enabled us to scan a volume whose dimensions were $73.8 \times 30 \times 34.5 \mathrm{~mm}$. Thus, the mesoscopic scale $L$ over which spatial averaging was performed was about 10 bead diameters. Importantly, measurements were performed between $1 \mathrm{~cm}$ to $4 \mathrm{~cm}$ away from the sidewall to neglect the side boundary layer effects as observed in Figure 3. Uniformity and repeatability were examined in Rousseau (2019) and in Rousseau \& Ancey (2020), which provide further information on the experimental procedure and findings.

\subsection{The vertical origin definition: the crucial role of bed arrangement}

To compare velocity and porosity profiles, we first needed to set the origin of the vertical axis. There is no standard procedure for doing this with rough beds. We examined various possibilities. The roughness crest - generally estimated by $z_{r c} \sim z_{\epsilon=0.99}$, as suggested by Pokrajak et al. (2006)) - was unsuitable because it created significant scatter between profiles when bed was rearranged. Indeed, $z_{\epsilon=0.99}$ was highly influenced by individual grains that were slightly higher than the average bed level. The origin had to be fixed at a bed height where the scatter between the porosity profiles was minimal. We found $z_{\epsilon=0.8}$ (see Figure 4 and Figure 4.12 in Rousseau (2019)), that is, at approximately $0.3 d_{p}$ below the roughness crest, which was the shift that Voermans et al. (2017) obtained using the porosity inflection method, and which was close to what Nezu (1993) prescribed. The Reynolds numbers based on this choice were slightly different from those estimated from the height of the roughness crest, as the flow depth $h_{f}$ was computed from $z_{\epsilon=0.8}$, which was above $z_{\epsilon=0.99}$.

\section{MODELING}

\subsection{Double-averaged momentum equation}

Double-averaging the Navier-Stokes equations produces the double-averaged momentum equations, whose projection on the streamwise $x$-direction for steady uniform flows is:

$$
0=\epsilon \rho_{f} g i+\frac{\mathrm{d} \tau_{d}}{\mathrm{~d} z}+\frac{\mathrm{d} \tau_{t}}{\mathrm{~d} z}+\frac{\mathrm{d} \tau_{v}}{\mathrm{~d} z}+f_{p, x}+f_{v, x},
$$

where $\left.\tau_{t}=-\rho_{f} \epsilon\left\langle\overline{u_{x}^{\prime} u_{z}^{\prime}}\right\rangle\right)$ and $\tau_{d}=-\rho_{f} \epsilon\left\langle\widetilde{u}_{x} \widetilde{u}_{z}\right\rangle$ are the turbulent and dispersive stresses respectively. The symbol ' $\bullet$ ' denotes the time averaged variable, the brackets ' $\langle\bullet\rangle$ ' indicate the intrinsic space averaged variable (i.e. over the fluid phase only). Equation 1 is obtained by employing the decomposition of the local instantaneous velocity $u_{i}=U_{i}+\widetilde{u}_{i}+u_{i}^{\prime}$ (in the $i$-direction) in term of temporal fluctuation $u_{i}^{\prime}$, the spatial disurbance $\widetilde{u}_{i}$ and the double-averaged velocity $U_{i}=\left\langle\overline{u_{i}}\right\rangle$ (see Nikora et al. (2007) for further details). $U_{x}$ is the averaged streamwise velocity and $U_{y} \sim 0$ 
to verify quasi-uniformity at the mesoscopic scale. $f_{p, x}$ and $f_{v, x}$ are called the pressure drag and viscous drag on the solid element surfaces. $\tau_{v}=\nu \mathrm{d}^{2} U_{x} / \mathrm{d} z^{2}$ is the viscous stress.

\subsection{Closure choices}

Following Prandtl's mixing-length approach, we express the turbulent stress by:

$$
\tau_{t}=\rho_{f} \epsilon l_{m}^{2}\left(\frac{\mathrm{d} U_{x}}{\mathrm{~d} z}\right)^{2}
$$

The damping effect, introduced by van Driest (1956) and documented by Nezu \& Rodi (1986) in open channel flows, is expected to play an active role for our experimental conditions. Indeed, for intermediate Reynolds numbers, i.e. $R e=O(10-1000)$, the damping effect reduces the vertical momentum transfer by turbulence in near-bed regions. Unlike van Driest (1956) who stated that roughness is expected to make the damping effect negligible, our results strongly suggest that this effect remains important for rough beds as soon as Reynolds numbers are sufficiently low. Thus, based on the original van Driest damping formulation (van Driest 1956), we express $l_{m}$ by:

$$
l_{m}=\kappa Z\left(1-\exp \left(-\frac{\sqrt{Z_{v D} U_{x}(z) / \nu}}{R e_{v D}}\right)\right)+C_{v D} l_{p}
$$

where $\kappa$ denotes the van Kármán "constant" that is fixed at $\kappa=0.41 . R e_{v D}$ and $C_{v D}$ are two constants that must be provided. $l_{p}$ is the pore length scale inside the bed given by the ratio of the volume of voids $V_{v}$ to their surface area $A_{v}, l_{p}=V_{v} / A_{v}=\epsilon_{b} d_{p} /\left(6-6 \epsilon_{b}\right)$. Within the van Driest approach, the value of the damping factor $1-\exp \left(-\sqrt{Z U_{x}(z) / \nu} / R e_{v D}\right)$ is given by relating the local Reynolds number $Z U_{x}(z) / \nu$ to $R e_{v D}$ with $Z$ the distance from the bed.

The definition of $Z$ must be clarified for rough bed and the integrating procedure is suggested:

$$
Z=\int_{-\infty}^{z} \sqrt{\frac{\epsilon(z)-\epsilon_{b}}{1-\epsilon_{b}}} d z
$$

The integration approach has the principal advantage to discard the origin definition ambiguity from the model. For the drag forces exerted on the porous bed, i.e. for $\epsilon(z)<1$ below the roughness crest, we employed the Ergun (1952) relation which consider pressure and viscous energy looses:

$$
f_{v, x}=-180 \frac{(1-\epsilon)^{2} \nu \rho_{f}}{\epsilon d_{p}^{2}} U_{x}, \quad f_{p, x}=-1.75 \frac{(1-\epsilon)}{d_{p}} \rho_{f} U_{x}^{2}
$$

Note that $f_{v, x}$ is the Kozeny-Carman term that is dominant for low velocites in porous medium. From mechanistic arguments, the following expression for the dispersive stress is suggested:

$$
\tau_{d}=1 / 2 \rho_{f} \epsilon \lambda_{+}\left(\frac{1-\epsilon(z)}{1-\epsilon_{b}}\right)^{3 / 2} U_{x}^{2}(z)
$$

where $\lambda_{+}$is the fraction of the fluid volume at a vertical coordinate where the local cross product $-\tilde{u}_{x} \tilde{u}_{z}$ is positive. For conciseness, we only summarized our choice here. Chapter 3 in Rousseau (2019) is devoted to this closure problem where terms for equations (2 - 6) are discussed.

\section{RESULTS}

\subsection{Hydraulic conditions and vertical structure}

In this section we report the results regarding two experimental runs with the same flow discharge per unit width $\left(q_{f}=0.30 \pm 0.015 \mathrm{~m}^{2} / \mathrm{s}\right)$. Hydraulic characteristics are detailed in Table 2 and double-averaged profiles are shown in Figure 4. Fully turbulent flows were observed when experiments were performed at intermediate Reynolds numbers, i.e., $R e=O(1000)$. Thus, turbulent stress $\tau_{t}$ is the dominant mechanisms for transferring momentum in the surface layer and follows the trend of the gravity force integrated between $z_{\text {surf }}$ and $z$, i.e. defined by : $G=-\int_{z}^{z_{\text {surf }}} \epsilon(z) \rho_{f} g i \mathrm{~d} z$ wich is equal to $\left(z-z_{\text {surf }}\right) \rho_{f} g i$ above $z_{r c} . \tau_{t}$ has its maximum at the roughness crest and is damped inside the roughness layer. 

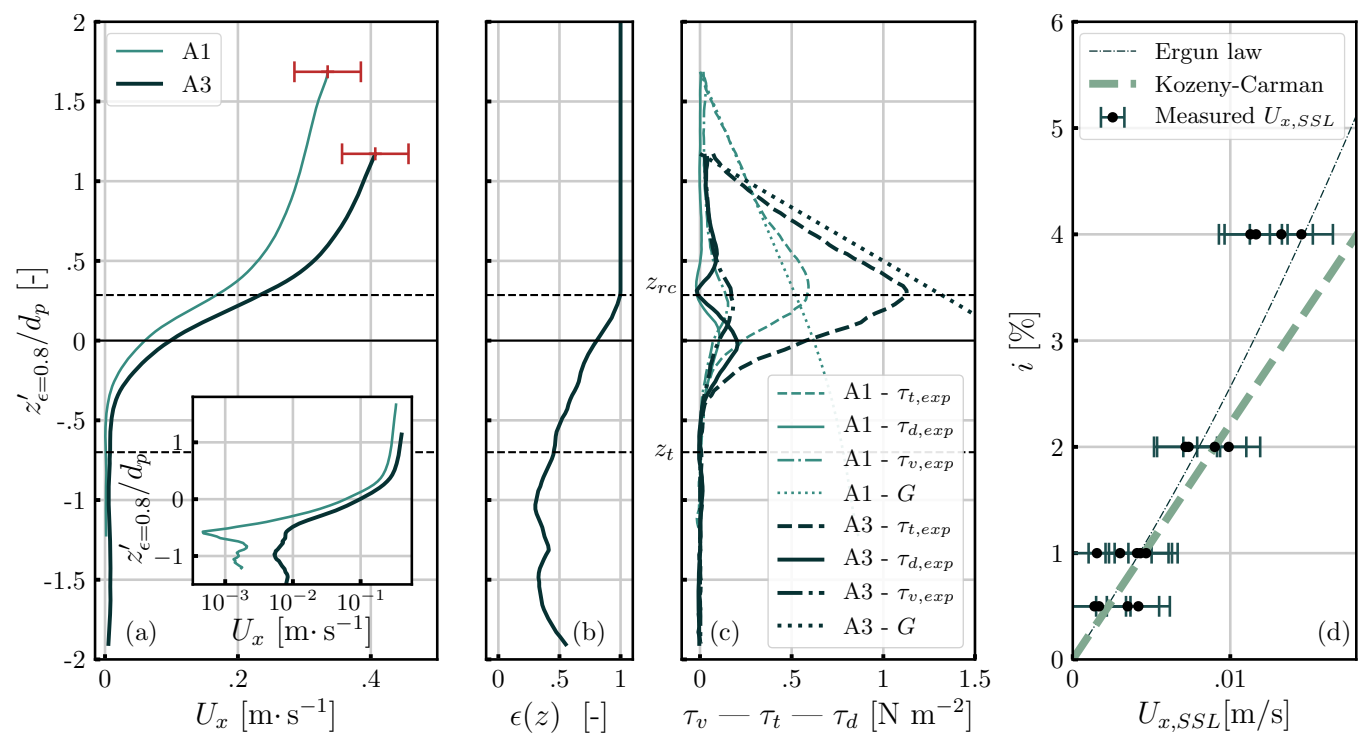

Figure 4. Vertical profiles for A1 $(i=0.5 \%)$ and A3 $(i=2 \%)$ runs with a constant flow discharge. (a) Velocity profiles: small velocities are shown with a logarithmic scale on the abscissa. The thin horizontal error bars represent uncertainties at free surface level. (b) Porosity profile. (c) Stress compared with $G$. The vertical coordinate is normalized by the mass-median-diameter $d_{p}$ and defined by $z_{\epsilon=0.8}^{\prime}=z-z_{\epsilon=0.8}$. (d) Plot of measured $U_{x, S S L}$ values that represents subsurface velocities below $z_{t}$ for 17 trials with various slopes. [Thin line] Ergun prediction, i.e. by solving $f_{v, x}\left(U_{x}\right)+f_{p, x}\left(U_{x}\right)=\epsilon_{b} \rho g i$ with $f_{v, x}\left(U_{x}\right)$ and $f_{p, x}\left(U_{x}\right)$ given by equation (5). [Thick line] Kozeny-Carman prediction, i.e. by solving $f_{v, x}\left(U_{x}\right)=\epsilon_{b} \rho g i$

Table 2. Hydraulic parameters of two runs

\begin{tabular}{lllllllll}
\hline Run & $i(\%)$ & $h_{f}[\mathrm{~mm}]$ & $U_{b}[\mathrm{~m} / \mathrm{s}]$ & $u_{*}[\mathrm{~mm} / \mathrm{s}]$ & $\operatorname{Re}_{b}$ & $F r^{*}$ & $R e_{K}^{* *}$ & Sh \\
\hline A1 & 0.5 & 13 & 0.26 & 26 & 1147 & 0.92 & 2.2 & 0.007 \\
A3 & 2.0 & 10 & 0.32 & 45 & 1095 & 1.29 & 3.8 & 0.019 \\
\hline
\end{tabular}

* Fr $=U_{\text {surf }} / \sqrt{g h_{f}}$ is the Froude number

** $R e_{K}=\frac{\sqrt{K_{K C}} u_{*}}{\nu}$ is the permeability Reynolds number as defined by Voermans et al. (2017) with

$K_{K C}=\frac{\epsilon_{b}^{3}}{180\left(1-\epsilon_{b}\right)^{2}} d_{p}^{2}$ the Kozeny-Carman equivalent permeability

The dispersive stress $\tau_{d}$ has its maximum at the vertical coordinate $z_{\epsilon=0.8}$ with a maximum beeing $5 \%$ of $\tau_{t}$ maximum. These vertical structures are in agreement with Voermans et al. (2018) observations although our experiments where performed at higher slopes and lower submergence values. Owing to slope variability, subsurface velocities are affected. Influence of gravity on the fluid in the subsurface layer corresponds to Ergun law predictions (see Figure $4-(d)$ ).

\subsection{Measured and modeled profiles}

Equation (1) with closures $(2-6)$ constitute the 1D model describing the vertical flow structure. We solve this system with a finite difference technique by employing the numerical scheme presented in Rousseau (2019) - Annex B. Simulated profiles were computed with hydraulic conditions given in Tables 1 and 2 for A 3 run. In the model, the porosity profile $\epsilon(z)$ is required. These profiles can be deduced from experimental work or estimated with a synthetic $\epsilon(z)$. The first solution has been chosen as we collected $\epsilon(z)$ from the PIV-RIMS procedure. For predicting flows in real river, assuming a synthetic profiles may be required (we use this solution in the next section). Figure 5 shows a comparisions between experimental profiles and the simulated profiles. Three parameters have been adjusted $\lambda_{+}=0.3, R e_{v D}=70$ and $C_{v D}=0.5$. Note that this parametrization has been tested with slopes ranging from 0.5 to $8 \%$ in Rousseau (2019). 

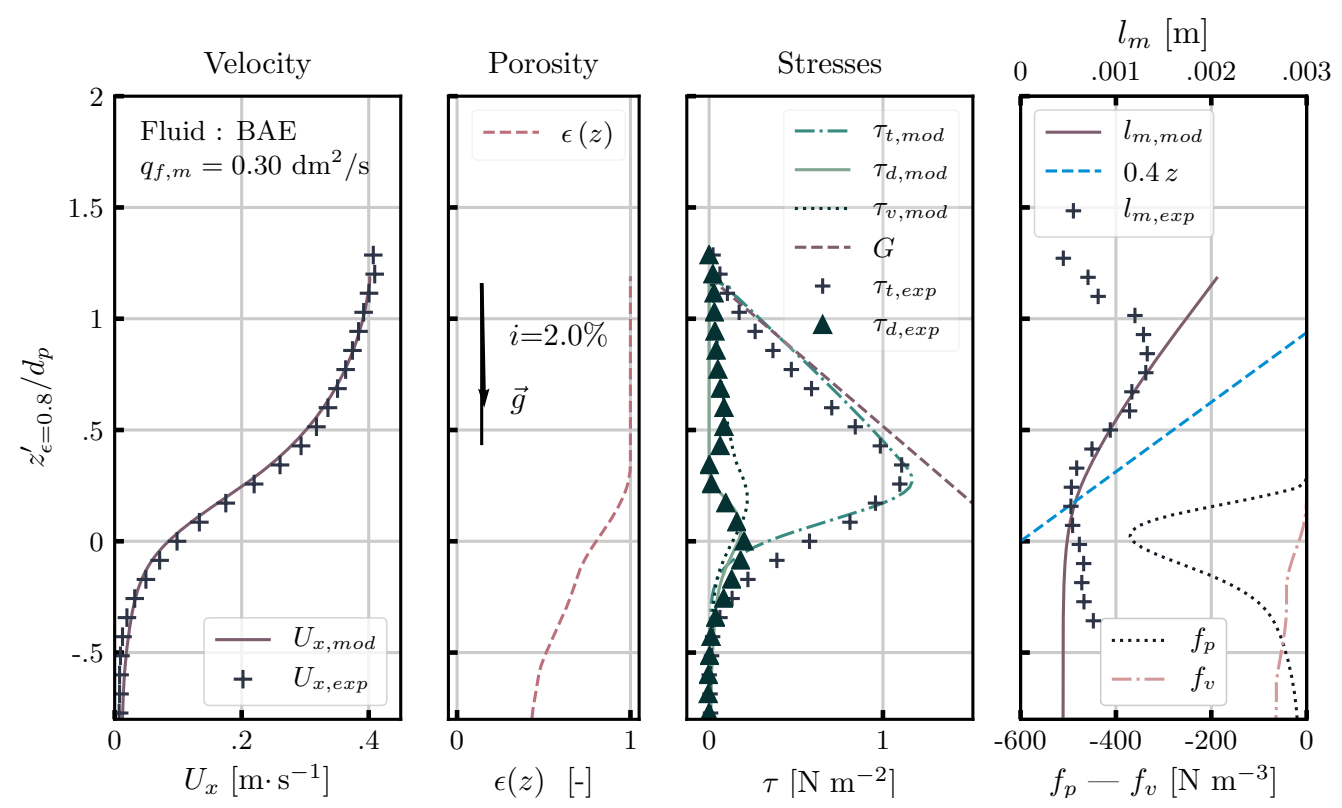

Figure 5. Simulated and measured double-averaged profiles for A3 run. Vertical profiles of (a) velocity, (b) porosity, (c) turbulent $\tau_{t}$, dispersive $\tau_{d}$ and viscous $\tau_{v}$ stresses and (d) pressure and viscous drag forces. Experimental and simulated $l_{m}$ is ploted with reference axis at the top. Experimental $l_{m}$ points are deduced by reversing equation (2) with the knowledge of $\tau_{t, \exp }$ and $U_{x, \exp }$.
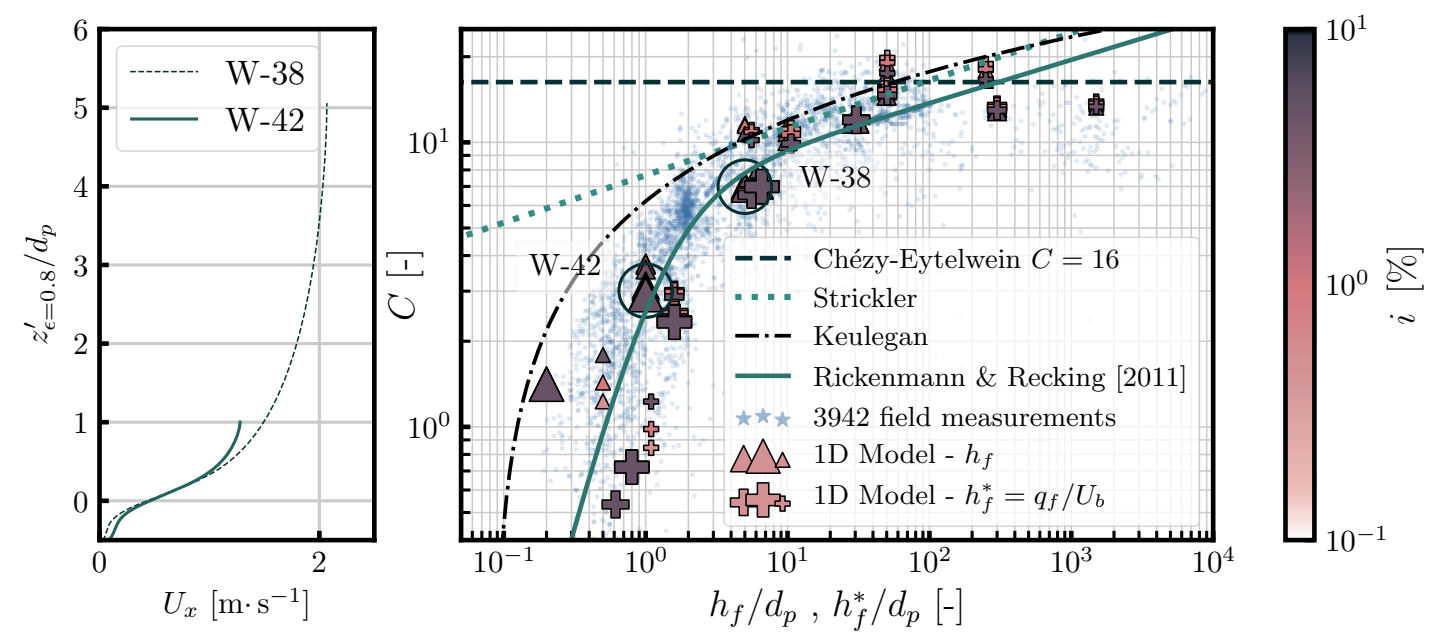

Figure 6. [Left] Velocity profiles W-38 and W-42 computed with the 1D model and real-world stream conditions - W-31 $\left(d_{p}=0.1 \mathrm{~m}, i=1 \%, h_{f}=0.5 \mathrm{~m}\right)$ and W-34 $\left(d_{p}=0.1 \mathrm{~m}, i=5 \%\right.$, $\left.h_{f}=0.1 \mathrm{~m}\right)$. [Right] Computation of 48 combinations in terms of the non-dimensional Chézy coefficient $C=U_{b} / \sqrt{g h_{f}} i$ versus relative submergence. Combinations W-31 and W-34 have been positioned on the plot. Symbols size depends on grain-size, and color is related to bed slope. The equations selected for the comparisons are: the Chézy-Eytelwein equation $C=16$, Strickler equation $C=24\left(h_{f} / d_{p}\right)^{1 / 6} / g^{1 / 2}$, Keulegan equation $C=1 / 0.4 \ln \left(12 * h / d_{p}\right)$, and the Rickenman \& Recking's (2011) relation $C=$ $4.41\left(h_{f} / d_{p}\right)^{1.9}\left(1+\left(h_{f} / d_{p} / 1.3\right)^{1.6}\right)^{-1.08}$ which is calibrated on 2890 selected data from a large data set of 3942 field measurements (tiny blue stars).

\subsection{Depth averaged simulated profiles and flow resistance}

The above mentioned simulations and experiments were performed at $R e=O(1000)$. These values are two orders of magnitude lower than typical Reynolds numbers for gravel bed rivers. Establishing if such modeling assumptions are transferable to real-world streams is important. As a first step, the comparison can be done by employing the following procedure: (i) simulating profiles with the 1D model for higher Re, i.e. with water, real hydraulic conditions and with the above 
calibrated parameters $C_{v D}, R e_{v D}$ and $\lambda_{+}$, (ii) averaging profiles over depth to deduce $U_{b}$ and the non-dimensional Chézy coefficient $C=U_{b} / \sqrt{g h_{f}}$, (iii) comparing $C$ with well-known flow resistance laws used in steep rivers. Simulations were performed with $d p=[0.002,0.01,0.1,0.5]$ $\mathrm{m}, h_{f}=[0.001,0.01,0.1,0.5,3] \mathrm{m}$, and $i=[0.5,1,5] \%$. All possible combinations result in 60 simulations producing different relative submergence conditions. As explained above, with low relative submergence, flow depth definition is crucial. We used two definitions: (i) as above, the flow depth defined by $h_{f}=z_{\text {surf }}-z_{\epsilon=0.8}$, and (ii) a flow depth estimated from the total flow discharge from mass conservation $h_{f}^{*}=q_{f} / U_{b}$. This alternative definitions is common in field studies since flow discharge is more accurately monitored than flow depth in mountain rivers (Recking, pers. comm.). The non-dimensional Chézy coefficient $C$ is plotted against the relative submergence $h_{f} / d_{p}$ in Figure 6. Interestingly, the resistance values computed with the present model and the classic equations provide results with the same order of magnitude and similar trends. It confirms that under various hydraulic conditions, our model is consistent with extant empirical equations. For high relative submergence flows $\left(h_{f} / d_{p}>10\right)$, computed points do not show substantial sensibility to the flow depth definitions. This is a consequence that $d_{p}$ is negligible with respect to $h_{f}$. For low relative submergence conditions $\left(h_{f} / d_{p}<10\right)$, variability is more important, reflecting the importance of the flow depth definition. It comes as no surprise to see that the curve given by Rickenman \& Recking (2011) is located between the $h_{f}$ and $q_{f} / U_{b}$ points. Indeed, this equation was fitted to field data where various definitions for flow depth are applied.

\section{CONCLUSION}

This proceeding presented a model able to reproduce the vertical structure of turbulent flows over rough permeable beds in low relative submergence conditions. This model was successfully compared to experimental profiles collected at intermediate Reynolds numbers $(R e=O(1000))$. In this condition, we revealed the role played by the damping effect on mixing length. While laboratory experiments were performed at Reynolds numbers two orders of magnitude lower than those of regular streams, computed flow resistance deduced from vertical profiles were successfully compared to flow resistance equation employed in river studies. A next step would be to identify the negligible terms in the 1D model in order to derive a flow resistance law for rivers.

\section{REFERENCES}

van Driest, E. R. 1956. On turbulent flow near a wall. Journal of the Aeronautical Sciences. 23 (11), 1007-1011.

Ergun, S., 1952. Fluid flow through packed columns. Chem. Eng. Prog. 48, 89-94.

Mignot, E., Barthelemy, E., \& Hurther, D. 2009. Double-averaging analysis and local flow characterization of near-bed turbulence in gravel-bed channel flows. Journal of Fluid Mechanics 618: 279-303.

Nezu, I. \& Rodi, W. 1986. Open-channel flow measurements with a laser doppler anemometer. Journal of Hydraulic Engineering. 112 (5), 335-355.

Nezu, I. \& Nakagawa H., 1993. Turbulence in open-channel flows. IAHR-monograph.

Ni, W.J. \& Capart, H., 2015. Cross-sectional imaging of refractive-index-matched liquid-granular flows. Experiments in Fluids, 56(8), p.163.

Nikora, V., McEwan, I., McLean, S., Coleman, S., Pokrajac, D. \& Walters, R., 2007. Double-averaging concept for rough-bed open-channel and overland flows: Theoretical background. Journal of Hydraulic Engineering, 133(8), pp.873-883.

Pokrajac D., Finnigan J.J., Manes C., McEwan I. \& Nikora V., 2006. On the definition of the shear velocity in rough bed open channel flows. Proc. of the International Conference on Fluvial Hydraulics, "RiverFlow 2006", Vol. 1: pp. 89-98.

Rickenmann, D. \& Recking, A., 2011. Evaluation of flow resistance in gravel-bed rivers through a large field data set. Water Resources Research, 47(7).

Rousseau G., 2019. Turbulent flows over rough permeable beds in mountain rivers: Experimental insights and modeling. PhD thesis, École Polytechnique Fédéral de Lausanne.

Rousseau G. \& Ancey C., 2020. Scanning PIV of turbulent flows over rough permeable beds using refractive index matching. In revision in Experiments in Fluids.

Voermans, J.J., Ghisalberti, M. \& Ivey, G.N., 2017. The variation of flow and turbulence across the sediment-water interface. Journal of Fluid Mechanics, 824, p.413. 